\title{
ALGEBRAIC MONOIDS WHOSE NONUNITS ARE PRODUCTS OF IDEMPOTENTS
}

\author{
MOHAN S. PUTCHA \\ (Communicated by Bhama Srinivasan)
}

\begin{abstract}
Let $M$ be a connected regular linear algebraic monoid with zero and group of units $G$. Suppose $G$ is nearly simple, i.e. the center of $G$ is one dimensional and the derived group $G^{\prime}$ is a simple algebraic group. Then it is shown that $S=M \backslash G$ is an idempotent generated semigroup. If $M$ has a unique nonzero minimal ideal, the converse is also proved. It follows that if $G_{0}$ is any simple algebraic group defined over an algebraically closed field $K$ and if $\Phi: G_{0} \rightarrow G L(n, K)$ is any representation of $G_{0}$, then the nonunits of the monoid $M(\Phi)=\overline{K \Phi\left(G_{0}\right)}$ form an idempotent generated semigroup.
\end{abstract}

It has been shown by J. Erdos [3] (see also [2]) that any nonsingular matrix over a field is a product of idempotent matrices. Let $K$ be an algebraically closed field. Our interest is in connected linear algebraic monoids with zero. This means by definition that the underlying set is an irreducible affine variety and that the product map is a morphism (i.e. a polynomial map). Let $G$ denote the group of units of $M, S=M \backslash G$. We are interested in knowing when $S$ is idempotent generated. We will only consider the situation when $G$ is a reductive group. This means $[\mathbf{1}]$ that the unipotent radical of $G$ is trivial. Then by $[\mathbf{8}, \mathbf{1 0}], M$ is unit regular, i.e. $M=E(M) G$ where $E=E(M)=\left\{e \in M \mid e^{2}=e\right\}$. If $X \subseteq M$, let $E(X)=X \cap E(M)$ and $\langle X\rangle$ the semigroup generated by $X$. Let $\mathscr{J}, \mathscr{R}, \mathscr{L}, \mathscr{H}$ denote the usual Green's relations on $M$ (see [5]). If $a, b \in M$, then $a \mathscr{J} b$ means $M a M=M b M, a \mathscr{R} b$ means $a M=b M, a \mathscr{L} b$ means $M a=M b, \mathscr{H}=\mathscr{R} \cap \mathscr{L}$. The following result was proved by the author [9, Theorem 2.7].

THEOREM 1 [9]. Suppose $S=M \backslash G$ has a maximum $\mathcal{J}$-class (i.e. $S$ is an irreducible variety). Then $S$ is an idempotent generated semigroup.

Let $R, G^{\prime}$ denote the radical, derived group of $G$, respectively. Then $[1, \S 1.8], R$ is contained in the center of $G, G^{\prime}$ is semisimple and $G=R G^{\prime}$. Suppose $\operatorname{dim} R>1$. Then there exists $e \in E(\bar{R}), e \neq 0,1$. There exists $g \in G$ such that $e g \neq e$. If $e g \in\langle E(M)\rangle$, then $e g=e_{1} \cdots e_{k}$ for some $e_{i} \in E(M), i=1, \ldots, k$. Then since $e$ is a central idempotent, $e g=e_{1}^{\prime} \cdots e_{k}^{\prime}$, where $e_{i}^{\prime}=e e_{i} \in E(M)$ and $e_{i}^{\prime} \leq e$. This is a contradiction since $M$ is a matrix semigroup. Thus $S=M \backslash G$ is not idempotent generated. We will say that $G$ is nearly simple if $\operatorname{dim} R=1$ and $G^{\prime}$ is a simple algebraic group, i.e. $G^{\prime}$ has no nontrivial closed connected normal subgroups.

THEOREM 2. Suppose $G$ is nearly simple. Then $S=M \backslash G$ is an idempotent generated semigroup.

Received by the editors February 1, 1987.

1980 Mathematics Subject (Classification (1985 Revision). Primary 20G99, 20M10. 
Proof. Let $R$ denote the radical of $G, G^{\prime}$ the derived group of $G$. Since $\operatorname{dim} R=1$, we see that $E(\bar{R})=\{0,1\}$. Also, in any faithful linear representation of $M$, every element of $G^{\prime}$ has determinant 1 . It follows that $G^{\prime}$ is closed in $M$. Let $e \in E=E(M), e \neq 0,1$. Let $G_{e}$ denote the connected component of the group $\{g \in G \mid g e=e g=e\}$. Then by [5],e $\in \bar{G}_{e}$. Let $G_{1}$ denote the subgroup of $G$ generated by $x^{-1} G_{e} x(x \in G)$. Then $G_{1}$ is a closed connected normal subgroup of $G, e \in \bar{G}_{1}$. So $G_{1} \nsubseteq R, G_{1} \nsubseteq G^{\prime}$. Since $G$ is nearly simple, we see that $G_{1}=G$. Let $E_{0}=\langle E(J)\rangle$,

$$
H=\left\{g \in G \mid e g \in\left\langle E_{0}\right\rangle\right\} .
$$

Let $f \in E_{0}, g \in H$. By [6, Lemma 1.12], there exist $e_{1}, f_{1} \in E_{0}$ such that $e \mathscr{L}$ $e_{1} \mathscr{R} f_{1} \mathscr{L} f$. Then $e_{1} g=e_{1}(e g) \in\left\langle E_{0}\right\rangle$. So $g e_{1}=g\left(e_{1} g\right) g^{-1} \in\left\langle E_{0}\right\rangle$. Hence $g f_{1}=\left(g e_{1}\right) f_{1} \in\left\langle E_{0}\right\rangle$. So $f_{1} g=g^{-1}\left(g f_{1}\right) g \in\left\langle E_{0}\right\rangle$. Thus $f g=f\left(f_{1} g\right) \in\left\langle E_{0}\right\rangle$. It follows that $E_{0} H \subseteq\left\langle E_{0}\right\rangle$. In particular $x^{-1} H x=H$ for all $x \in G$. Now let $a, b \in H$. Then $e a, e b \in\left\langle E_{0}\right\rangle$. So $b e=b(e b) b^{-1} \in\left\langle E_{0}\right\rangle$. Thus bea $=(b e)(e a) \in\left\langle E_{0}\right\rangle$. Hence $e a b=b^{-1}(b e a) b \in\left\langle E_{0}\right\rangle$. Thus $a b \in H$. Since $G_{e} \subseteq H$, we see that $G=G_{1} \subseteq H$. Hence $H=G$. Since $M$ is unit regular, we see that $S \subseteq\langle E\rangle$.

COROLlARY 1. Let $G_{0}$ be a simple algebraic group, $\Phi: G_{0} \rightarrow G L(n, K)$ any representation. Let $M(\Phi)$ denote the Zariski closure of $K \Phi\left(G_{0}\right)$ in $\mathscr{M}_{n}(K)$. Then the nonunits of $M(\Phi)$ form an idempotent generated semigroup.

We now consider the situation dual to that in Theorem 1, namely when $M \backslash\{0\}$ has a minimum $\mathcal{J}$-class. Equivalently, the intersection of all nonzero ideals of $M$ is again nonzero. Renner [11, Corollary 8.3.3] has characterized these monoids as being exactly those having an idempotent separating (equivalently finite in the algebraic geometry sense) irreducible, linear representation. Moreover, in such a case the radical $R$ of $G$ is one dimensional [11, Lemma 8.3.2].

THEOREM 3. Suppose $M$ has a unique nonzero minimal ideal. Then $S=M \backslash G$ is an idempotent generated semigroup if and only if $G$ is nearly simple.

PROOF. Let $R$ denote the one dimensional radical of $G$ and $J_{0}$ the nonzero minimum $\mathcal{J}$-class of $M$. So $J_{0} \cup\{0\}$ is the minimum nonzero ideal of $M$. If $G$ is nearly simple, then by Theorem $2, S$ is idempotent generated. Conversely suppose that $S \subseteq\langle E\rangle$ where $E=E(M)$. Suppose $G$ is not nearly simple. Then $G=G_{1} G_{2}$ where $G_{1}, G_{2}$ are proper closed connected normal subgroups of $G$ containing $R$, and such that $g_{1} g_{2}=g_{2} g_{1}$ for all $g_{i} \in G_{i}, i=1,2$. Let $M_{i}$ denote the closure of $G_{i}$ in $M, i=1,2$. Since $R \neq G_{1}$, there exists $e \in E\left(M_{1}\right), e \neq 0,1$. By [5], $J=G e G$ is the $\mathcal{J}$-class of $e$ in $M$. By [5], any element of $E(J)$ is conjugate to $e$. Since $G_{2}$ centralizes $M_{1}$ and $G=G_{1} G_{2}$, we see that $E(J) \subseteq M_{1}$. Let $g \in G, a=e g$. Then $a=e_{1} \ldots e_{m}$ for some $e_{1}, \ldots, e_{m} \in E$. Let

$$
e_{i}^{\prime}=e_{i} \cdots e_{m} g^{-1} e e_{1} \cdots e_{i} \in E(J), \quad i=1, \ldots, m .
$$

Then with $e_{0}=e$, we have for $i=1, \ldots, m$,

$$
e_{0} \cdots e_{i-1} e_{i}^{\prime}=e_{0} \cdots e_{m} g^{-1} e e_{1} \cdots e_{i}=e g g^{-1} e e_{1} \cdots e_{i}=e e_{1} \cdots e_{i}
$$

So $e e_{1}^{\prime} \cdots e_{m}^{\prime}=e e_{1} \cdots e_{m}=a$. Thus $a \in\langle E(J)\rangle \subseteq M_{1}$. Hence $g e=g a g^{-1} \in$ $\langle E(J)\rangle \subseteq M_{1}$. It follows that $J=G e G \subseteq M_{1}$. Hence $M e M=\bar{J} \subseteq M_{1}$. Thus $J_{0} \subseteq M_{1}$. So $J_{0}$ is centralized by $G_{2}$. Similarly $J_{0} \subseteq M_{2}$ and $J_{0}$ is centralized by 
$G_{1}$. Thus $J_{0}$ is centralized by $G$. So if $f \in E\left(J_{0}\right)$, then by [7, Theorem 2.3], $f \in \bar{R}$. This contradicts the fact that $\operatorname{dim} R=1$, proving the theorem.

The difference between Theorems 1 and 3 is illustrated by the following elementary examples.

EXAMPLE 1. Let $M=\left\{A \oplus B \mid A, B \in \mathscr{M}_{2}(K), \operatorname{det} A=\operatorname{det} B\right\}, G$ the group of units of $M$. Then $S=M \backslash G$ has a maximum $\mathscr{J}$-class and by Theorem $1, S$ is idempotent generated. Note that $G$ is not nearly simple.

EXAMPLE 2. Let $M=\left\{A \otimes B \mid A, B \in \mathscr{M}_{2}(K)\right\}, G$ the group of units of $M$, $S=M \backslash G$. Then $M$ has a unique nonzero minimal ideal. Clearly $G$ is not nearly simple and hence by Theorem $3, S$ is not idempotent generated.

\section{REFERENCES}

1. R. W. Carter, Finite groups of Lie type: conjugacy classes and complex characters, Wiley, 1985.

2. R. J. H. Dawlings, On idempotent affine mappings, Proc. Roy. Soc. Edinburgh Sect. A 93 (1983), 345-348.

3. J. A. Erdos, On products of idempotent matrices, Glasgow Math. J. 8 (1967), 118-122.

4. J. M. Howie, An introduction to semigroup theory, Academic Press, New York, 1976.

5. M. S. Putcha, Green's relations on a connected algebraic monoid, Linear and Multilinear Algebra 12 (1982), 205 214.

6. __ Connected algebraic monoids, Trans. Amer. Math. Soc. 272 (1982), 693-709.

7. __ A semigroup approach to linear algebraic groups, J. Algebra 80 (1983), 164-185.

8. __ Reductive groups and regular semigroups, Semigroup Forum 30 (1984), 253-261.

9. ___ Regular linear algebraic monoids, Trans. Amer. Math. Soc. 290 (1985), 615-626.

10. L. E. Renner, Reductive monoids are von-Neumann regular, J. Algebra 93 (1985), 237-245.

11. __ Classification of semisimple algebraic monoids, Trans. Amer. Math. Soc. 292 (1985), 193-224.

Department of Mathematics, North Carolina State University, Raleigh, NORTH CAROLINA 27695-8205 